\title{
From Infancy to Aging: Biological and Behavioral Modifiers of Fetuin-A
}

\author{
Katie Robinson, MPH ${ }^{1,2}$ \\ Margarita Teran-Garcia, MD, Ph.D. ${ }^{1,2,3}$
}

${ }^{1}$ Illinois Transdisciplinary Obesity Prevention Program

${ }^{2}$ Division of Nutritional Sciences

${ }^{3}$ Department of Food Science and Human Nutrition University of Illinois at Urbana-Champaign

437 Bevier Hall, MC-182

905 S Goodwin Avenue

Urbana, Illinois 61801 USA

Ph: (217) 244-2025

Fax: (217) 265-0925

teranmd@illinois.edu 


\begin{abstract}
Fetuin-A is a multifunctional protein which inhibits ectopic calcification and insulin receptor phosphorylation. It may also promote insulin resistance by activation of toll-like receptor 4 proinflammatory cascade. Increased levels of Fetuin-A have been associated with obesity and related comorbidities such as type 2 diabetes and cardiovascular disease, therefore, it has been suggested as a potential therapeutic target for intervention. However, in addition to its role in disease pathophysiology, Fetuin-A is also important for growth and development. Thus, before evaluating Fetuin-A as a biomarker or pharmacological target, an understanding of Fetuin-A variability throughout the life cycle is essential. This manuscript reviews the current body of knowledge surrounding Fetuin-A throughout the life cycle and discuss challenges to studying Fetuin-A.
\end{abstract}

\title{
Keywords
}

Fetuin-A, alpha2-Heremans Schmid glycoprotein, AHSG. 


\section{$\underline{\text { 1. Introduction }}$}

Fetuin-A, also known as alpha 2-Heremans Schmid glycoprotein (AHSG), was first recognized due to its high concentration in fetal serum [1]. Since being discovered, this endogenously synthesized protein has been ascribed multiple biological functions but the understanding of these mechanisms remains poor. Circulating levels of Fetuin-A have been associated with numerous chronic diseases; most notably, renal disease, cardiovascular disease (CVD) and type 2 diabetes (T2DM) [2-7].

\subsection{Structure and function}

Human Fetuin-A is mainly secreted from the liver, but many other tissues are able to express this protein (i.e. adipose, placenta and tongue) $[8,9]$. The structure of Fetuin-A, including other mammalian isoforms, has been described in great detail [10-12]. Briefly, Fetuin-A is secreted as a single chain precursor. This precursor is cleaved during proteolytic processing in the Golgi apparatus to form a heavy and a light chain consisting of 321 and 27 amino acids, respectively. A connecting peptide, which is 40 amino acids in length, is cleaved from the $\mathrm{C}$ terminus of the heavy chain $[13,14]$. The remaining chains are joined by six disulfide bonds. The final (mature) form of Fetuin-A has three domains. These domains contain important functional and structural features. The first domain contains a calcium-binding site. Domain 2 has three $\mathrm{N}$-linked glycosylation sites and a phosphorylation site at Ser120. The final domain (Domain 3) contains two O-linked glycosylation sites as well as the second phosphorylation site at Ser312 [11, 12, 15]. It is estimated that $20 \%$ of FetA is phosphorylated in at least one site and phosphorylation appears to be essential for activation of Fetuin-A $[11,13,15,16]$. 
The complex structure of Fetuin-A suggests multiple biological functions. Specifically, Fetuin-A encloses a TGF-beta binding site, in the first domain which allows to influence cell differentiation and proliferation $[17,18]$. Indeed, Fetuin-A is a natural antagonist of TGF-beta and bone morphogenetic protein (BMP) activities [17-19]. Fetuin-A also contains a calcium binding site which supports its proposed role as a mineral chaperone and inhibitor of calcification [20, 21]. The disulfide bonds, for apatite precipitation, and the TGF-beta/ BMP binding sites are integral to Fetuin-A's function in bone development and CVD [17, 18, 22].

Fetuin-A appears to be important in managing systemic calcification. Specifically, FetuinA reduces ectopic calcification that contributes to development of CVD [18, 20, 21, 23, 24]. Low Fetuin-A in circulation may lead to increased mortality risk [20]. However, high Fetuin-A levels may contribute to the pathophysiology of T2DM. Circulating Fetuin-A is higher in individuals with T2DM than those with normal glucose tolerance $[12,25,26]$. This may be explained by the finding that Fetuin-A diminishes insulin response in muscle and adipose cells by inhibiting autophosphorylation of the insulin receptor [12]. Domain 2 of Fetuin-A is structurally similar to tyrosine kinase thus may inhibit it $[11,27]$. It should be noted that there is some controversy about this particular mechanism due to inconsistent findings reported [12, 15, 28]. At the molecular level, the question still remains as to why Fetuin-A is elevated in those with T2DM.

Another potential mechanism of Fetuin-A function has been discovered in cell culture and animal models. Emerging evidence suggests that Fetuin-A plays an important role in the development of lipid-induced insulin resistance. Palmitate bound Fetuin-A activates toll-like receptor-4 (TLR4); thus, signaling the release of proinflammatory cytokines, contributing to decreased insulin sensitivity [29]. In obesity, Fetuin-A may also orchestrate macrophage migration 
and polarization in adipocytes by acting as a chemokine [30]. Due to these findings, Fetuin-A has been proposed as a therapeutic target to treat obesity and related comorbidities [4].

While the evidence to link Fetuin-A with obesity has increased in recent decades, there is also accumulating research in the area of inflammation [24, 31-38]. Not only in the sub-chronic inflammation associated with excess adiposity, but also in the acute inflammation resulting from infection and injury. Fetuin-A has a somewhat elusive role in inflammation depending on the instigating stimulus and has the potential to behave as both a negative and a positive acute phase protein (APP) (recently revised by [36]). Early reports by Lebreton and colleagues show that circulating values fell during acute infection, which led to its characterization as a negative APP [33]. This finding is supported by cell culture and animal studies showing Fetuin-A temporarily decreases with administration of LPS [34, 38]. In those models with lower Fetuin-A, it was possible to launch an early inflammatory response to infection. Paradoxically, mice with endotoxemia that received supplemental Fetuin-A exhibited greater survival capabilities than mice with endotoxemia that did not receive Fetuin-A [34]. This protective effect may be explained by Fetuin-A's ability to decrease the release of proinflammatory cytokines such as IL-1 and TNF, therefore reducing the late inflammatory response to sepsis mediated by high mobility group box1 (HMGB1) [31]. Fetuin-A is also essential for spermine to act as an anti-inflammatory agent [37, 38]. Taken together, these findings suggest Fetuin-A decreases in response to acute inflammation but increases in response to late inflammation resulting from infection. Fetuin-A has also been shown to act as a positive APP in response to inflammation caused by injuries such as cerebral ischemia in both humans and cattle $[24,32,36]$. In these situations, the initial injury stimulates the release of HMGB1, which if left unmanaged would trigger subsequent damage and inflammation. The presence of Fetuin-A is thought to decrease the inflammatory response and prevent further 
damage [36]. It should be noted that Fetuin-A is normally unable to cross the blood brain barrier (BBB), however, following instances of trauma, the BBB permeability is temporarily increased allowing for Fetuin-A to enter damaged cerebral tissue [35]. Therefore, the type and source of inflammation may be an important consideration when determining the relationship between Fetuin-A and the immune response. These findings also indicate that accurate measures of acute infection and illness are important and need documentation in any study evaluating Fetuin-A.

\subsection{Challenges in measuring Fetuin-A}

Since many conditions influence circulating Fetuin-A, it has been difficult to establish reference values and Fetuin-A's role in normal mammalian development [1, 39, 40]. Current data suggest that Fetuin-A is highest during infancy, declines into childhood and varies in adults [4143]. The variability of Fetuin-A in adulthood may be attributed to diverse health behaviors, disease states and weight status. For example, weight management strategies such as caloric restriction, aerobic exercise and weight loss surgeries have all been shown to reduce circulating Fetuin-A while overfeeding and obesity tend to increase its levels [43-46]. Multiple comprehensive reviews on Fetuin-A in unhealthy populations have been published, however, scarce data are available discussing the action of Fetuin-A over the lifespan in healthy populations [6, 47-49]. There are few reports on Fetuin-A during childhood. Most randomized control trials evaluating the response of Fetuin-A to health behavior interventions include only adult cohorts. Of the existing reports in children, most have methodological limitations such as wide age variation, cross-sectional study design, or include only children with compromised health conditions such as renal disease and nonalcoholic fatty liver disease (NAFLD) [50-52]. 
Another limitation to our understanding of Fetuin-A is the variability of measurement methods. Several companies offer Fetuin-A specific enzyme-linked immunosorbent assays (ELISA); however, inconsistencies exist among reported values [53]. In a study by Smith et al., commercial kits from Epitope ${ }^{\mathrm{TM}}$ and Biovendor ${ }^{\mathrm{TM}}$, yielded differing values for circulating FetuinA in normal populations. The reported Fetuin-A values were 2.2 higher when using the Epitope ${ }^{\mathrm{TM}}$ ELISA kit than when using the Biovendor ${ }^{\mathrm{TM}}$ ELISA kit. Smith and collaborators demonstrated that glycosylation status may explain, in part, the difference between the two commercial kits [53]. Therefore, it is possible that the degree of post-translational modification of Fetuin-A may influence measurement accuracy. In addition to glycosylation sites, as mentioned, at least two important phosphorylation sites have been reported in Fetuin-A at Ser120 and Ser312 [11]. The impact of phosphorylation status on Fetuin A measurement by ELISA is not known. However, the high variability in the reported circulating values of Fetuin-A may be partially explained by technical differences or methodological selection of the epitope used by commercial kits. The heterogeneity of published results and pre-analytical variability are major challenges to the use of Fetuin-a as a novel biomarker of metabolic disease. This area eagerly awaits further clinical investigation.

Despite years of research, there are no standardized reference values of Fetuin-A for infants, children or adults. It is also unclear which factors influence Fetuin-A levels at these life stages. Therefore, the objective of this review is to discuss the current understanding of Fetuin-A across the lifespan focusing on the impact of development, pregnancy and aging as well as modifiable life factors including diet, exercise, weight management and elective surgeries (Figure 1). A summary of relevant human studies that were published in English are presented. The search terms included Fetuin-A, alpha2-Heremans Schmid Glycoprotein, AHSG and terms related to the 
lifecycle (i.e. infant, child, adult, pregnancy, elderly) in three search engines (PubMed, Scopus and Elsevier) until October of 2015. Both authors were involved in the search and selection of the papers summarized here.

\section{Insert Figure 1 about here}

\section{$\underline{\text { 2. Fetuin-A during the Lifespan }}$}

\subsection{Gestation and Pregnancy}

Due to high concentrations of Fetuin-A in fetal serum, multiple functions related to development have been suggested [1,8]. Fetuin-A constitutes $\sim 25 \%$ of the non-collagenous protein fraction of mammalian bone [54]. Following collagen, Fetuin-A is one of the most abundant proteins in bone and its ability to bind calcium and other minerals supports the notion that it is essential for osteochondrogenesis $[21,54,55]$. A role for Fetuin-A in cell proliferation and differentiation has also been demonstrated [56].

During normal gestation, serum Fetuin-A increases with each trimester [57]. However, women with the highest Fetuin-A levels during pregnancy had newborns with lower average body weights, shorter average body lengths and smaller average head circumferences [57]. Therefore, there appears to be a physiological reason for Fetuin-A to increase for bone development but there may also be negative effects from values that are too high.

The rise in Fetuin-A may be correlated with the observed decrease in insulin sensitivity during pregnancy. Thus, it could be postulated that women with gestational diabetes mellitus (GDM) would have the highest values of Fetuin-A, however, conflicting results have been reported [57, 58]. In a study of 104 women, those with GDM had higher serum Fetuin-A than healthy 
women at the same stage of pregnancy [57]. Conversely, in a study of 20 pregnant women, plasma Fetuin-A in women with GDM and those with normal glucose tolerance did not differ at the $28^{\text {th }}$ week of gestation or 10-12 weeks after delivery [58]. How maternal diet, maternal weight status and breastfeeding effect Fetuin-A in the mother and infant is unknown. Further research is needed to identify the function and significance of Fetuin-A during pregnancy and whether modulations in maternal Fetuin-A can influence the growth and development of the infant.

\subsection{Birth}

Fetuin-A is frequently described as an important developmental protein because of its high concentrations in fetal circulation $[8,59]$. Infants have Fetuin-A levels greater than $1 \mathrm{mg} / \mathrm{mL}$, however, values vary based on measurement methods and length of gestation $[39,41]$. Two studies have shown infants born prematurely to have higher plasma Fetuin-A levels than those born at term [39, 41]. Conversely, a study of 19 fetuses following therapeutic abortion found that FetuinA increased from 20 weeks gestation to 38 weeks gestation although this trend was not significant [8]. Notably, these studies included small sample sizes and limited information on the demographics and coexisting health conditions of the participants. Thus, studies of larger cohorts with more information about the ethnicity, sex, and health status of the infants as well as maternal demographics are needed. New studies could provide important information about the normal/optimal levels of Fetuin-A in early development.

As stated previously, Fetuin-A has the ability to inhibit calcification plays a critical role in bone growth. Fetuin-A knockout mice have stunted long bones growth likely resulting from premature mineralization of growth plates [40]. This inhibitory effect has been also demonstrated in vitro $[20,60]$. In humans, the hypothesis that Fetuin-A may be linked to restricted growth was 
tested in a comparison of appropriate-for-gestational-age infants and intrauterine-growth-restricted (IUGR) infants and no difference was found [61]. However, infants with IUGR have different glycosylation patterns on Fetuin-A than full-term infants [62]. Thus, although circulating FetuinA may not differ, there may be important post-translational modifications occurring throughout life that impact Fetuin-A functions.

\subsection{Childhood}

To evaluate Fetuin-A throughout childhood, Häusler and collaborators collected blood from 96 healthy children ages 3 weeks to 17 years old. No significant difference was seen when the average Fetuin-A was compared across age groups, suggesting that age was not a predictor of circulating Fetuin-A. The mean serum Fetuin-A was $0.58( \pm 0.12) \mathrm{mg} / \mathrm{ml}$, about half that of infants [41]. The authors of this publication did not comment on ethnicity or pubertal status of these children and only measured the children cross-sectionally. Therefore, the utility of these values in creating a reference range is low. A similar study in 246 Caucasian children, ages 9 days to 18 years, found comparable Fetuin-A concentrations $(0.46 \pm 0.24 \mathrm{mg} / \mathrm{ml})$. This study concluded that age, sex and body mass index standard deviation scores (BMI-SDS) did not alter Fetuin-A [42]. Importantly, children in this study had normal BMI-SDS, so there was limited ability to detect differences in Fetuin-A related to obesity.

The association between childhood obesity and Fetuin-A concentrations was specifically studied by Reinehr and Roth [52]. Fetuin-A was not significantly different between normal weight and obese children, however, obese children with NAFLD had significantly higher circulating Fetuin-A $(0.35 \pm 0.07 \mathrm{mg} / \mathrm{mL})$ than obese children without NAFLD $(0.29 \pm 0.06 \mathrm{mg} / \mathrm{mL})$ [52] Waist circumference was also positively correlated with Fetuin-A [52]. Overall, this study suggests 
that metabolically unhealthy obesity but not metabolically healthy obesity is positively correlated to Fetuin-A. The arguments on metabolically healthy and unhealthy obesity and its progression to metabolic disease are an issue of heated debate (as reviewed by [63-66]) and the role of Fetuin-A has been underlined, especially by Stefan and colleagues [67]. Further, Stefan's team group suggested that Fetuin-A could have a role in the severity or progression of NAFLD [68]. In general, studies in the field of obesity would benefit from using more precise measures of body composition such as dual X-ray absorptiometry and bioelectrical impedance because correlations between Fetuin-A and weight status may not be appreciated if obesity is defined by height and weight alone. In childhood, it is unclear how dietary intake impacts circulating Fetuin-A. The study of Fetuin-A response to dietary intake and physical activity includes mostly adult cohorts with the exception of a one-year nutrition and physical activity education intervention with obese participants (ages 8-12 years old). Fetuin-A levels were compared between a group of children who achieved significant weight loss (as evidenced by a 0.5 reduction in BMI-SDS) and a group of children who did not change body weight after the intervention. A $10 \%$ reduction in Fetuin-A was observed in children who lost weight [52]. It is uncertain based on the study design whether changes in body composition, dietary intake, or physical activity regimen impacted serum FetuinA, therefore, further studies are warranted.

Genetic influences may also impact Fetuin-A levels and function. Notably, a meta-analysis by Elks and collaborators found that BMI heritability estimates were highest in younger children [69]. Whether the heritability of Fetuin-A level follows a similar trajectory remains to be reported. The gene which codes for human Fetuin-A is AHSG [13]. At this time, we are unaware of any research investigating the impact of AHSG genetic polymorphisms on obesity or associated comorbidities during childhood, however, genetic polymorphisms in Fetuin-A have been linked to 
an increased risk of T2DM, obesity, and CVD in adults [50, 70, 71]. The genomic locus of AHSG (3q27) was found to be a susceptible region for early-onset diabetes and thus, may support an important role for Fetuin-A in the progression of childhood obesity associated comorbidities [72].

\section{Factors influencing Fetuin-A during Adulthood}

\subsection{Genetics}

The AHSG gene is located in the $3 \mathrm{q} 27$ region, which has been identified as a T2DM and obesity susceptible region $[72,73]$. Individual genetic variation may impact circulating Fetuin-A levels, as well as risk for T2DM and obesity-related phenotypes. Single nucleotide polymorphisms (SNPs) in the AHSG gene correlate with plasma Fetuin-A [74, 75]. In a cohort of 2197 adults, the rs4917 polymorphism explained $21.2 \%$ of the variance in Fetuin-A with each C-allele increasing Fetuin-A by $0.035 \mathrm{mg} / \mathrm{mL}$. To date, four SNPs have been found to be associated with circulating Fetuin-A [74].

Although Fetuin-A levels have been positively associated with obesity, the correlation between AHSG SNPs and obesity parameters is inconsistent [48]. Multiple AHSG-SNPs (rs2248690, rs2077119, rs4831, rs4917 and rs11540663) were genotyped in 364 normal weight and obese women and no associations with BMI, percent body fat or waist circumferences were found [76]. Another group genotyped rs4917 in 157 patients post-myocardial infarction and a significant association with waist circumference and a borderline significant association with BMI was found $(\mathrm{p}=0.065)$ [71]. When analyzed under a dominant model, and excluding individuals with T2DM, it appeared that T-allele carriers of rs4917 (T/T or C/T) had significantly lower BMI and waist circumferences than non-T-allele carriers (C/C) [71]. In a group of 504 normal weight and obese Swedish men, the G-allele of rs2593813 was associated with leanness [70]. Three 
AHSG SNPs (rs2593813, rs4917 and rs4918) were constructed into haplotypes and certain variations of these haplotypes were more common in lean versus obese men [70]. Conversely, another research group used magnetic resonance imaging to assess regional body fat and was unable to show correlations between body fat and genetic variations in the AHSG gene [77]. Overall, it appears that certain SNPs in AHSG influence circulating Fetuin-A, but these SNPs are inconsistently associated with BMI. Furthermore, few of these SNPs have been linked to functional changes in Fetuin-A whether that be decreased binding affinity for insulin receptor, TLR4, palmitate or other ligands [71]. However, it could be possible that some of the non-functional individual genetic variants reported in the AHSG gene are in linkage-disequilibrium (associated) with unknown functional variants.

\subsection{Obesity}

Since Fetuin-A was found to inhibit the human insulin receptor at the tyrosine kinase level, it prompt interest to investigate its association with insulin resistance and obesity [2]. A recent review paper has covered this topic in great detail [48]. Overall, circulating Fetuin-A is positively associated with obesity and weight gain and negatively associated with weight loss following bariatric surgery $[44,78]$. In an observational study spanning five years, baseline Fetuin-A was positively associated with gain of visceral adipose tissue in older adults. More specifically, each $0.42 \mathrm{mg} / \mathrm{mL}$ increase in Fetuin-A led to a 5\% increase in visceral adipose tissue gain [79]. On the other hand, a 34\% decrease in BMI caused by Roux-en-Y gastric bypass (GBP) led to a $19 \%$ decrease in Fetuin-A 16 months after surgery [44]. In another study of bariatric surgery patients, Fetuin-A was $27 \%$ lower just three days after surgery when compared to Fetuin-A levels three 
days before surgery [78]. Therefore, Fetuin-A does appear to fluctuate with weight but it is unclear whether the change in Fetuin-A precedes weight loss or vice versa.

Medications which alleviate obesity-associated comorbidities may also impact circulating Fetuin-A. The thiazolidinedione, pioglitazone, is prescribed to patients with T2DM to increase insulin sensitivity and has been shown to decrease Fetuin-A expression in hepatocytes [80]. Another anti-diabetic drug, metformin, has also been studied and has shown inconsistent correlations with Fetuin-A [80-82]. Extended release niacin, used for the treatment of dyslipidemia, decreases serum Fetuin-A and phosphofetuin [83]. Finally, the glucocorticoid, dexamethasone, which is often prescribed to relieve inflammation, has been shown to increase Fetuin-A transcription through CCAAT enhancer binding protein-beta and hepatocyte nuclear factor 3-beta [84]. Therefore, future studies should note whether participating subjects are taking prescriptions which may modify circulating Fetuin-A.

\subsection{Diet}

High fat diets stimulate Fetuin-A transcription and Fetuin-A preferentially binds the saturated fatty acid, palmitate and signals the TLR4 inflammatory cascade [29, 30]. In vitro studies suggest that high carbohydrate and high fat environments promote the expression of Fetuin-A in hepatocytes and adipocytes and may result in higher circulating levels in vivo [30]. However, a recent study of a population in Bavaria $(\mathrm{n}=558)$ showed that circulating Fetuin-A was not associated with total energy or specific macronutrient intake as calculated from three 24 hour dietary recalls [85]. In order to avoid the bias of self-reported food intake, another study evaluated circulating free fatty acids and found a positive correlation with circulating Fetuin-A [6]. Although 
there was no consistent association with fat intake and Fetuin-A, it is unclear whether specific fatty acids preferentially stimulate the secretion or function of Fetuin-A.

Clinical studies (primarily investigating insulin resistance) have demonstrated effects on Fetuin-A resulting from dietary interventions, including: 1) overfeeding, 2) calorie restriction, and 3) modified intake of specific nutrients (for example: omega-3 polyunsaturated fatty acids (PUFA), caffeine and alcohol) $[43,86-90]$.

Plasma Fetuin-A was increased after overfeeding 40 healthy adults for 28-days [46]. The composition of their diet was increased by $1100 \mathrm{kcals} / \mathrm{d}$ from baseline and modified to be composed of $45 \%$ energy from fat, $15 \%$ energy from protein and $40 \%$ energy from carbohydrates. This short report did not provide numerical values for baseline and post-overfeeding circulating Fetuin-A, so the magnitude of influence is not known. Furthermore, no descriptive information about the specific fatty acid content, ratio of saturated fat to monounsaturated and polyunsaturated fatty acids or other nutrition details were reported. Therefore, although overfeeding seems to increase Fetuin-A, there is no conclusive evidence on whether the type of dietary fat increased during overfeeding matters.

Calorie restriction appears to reduce Fetuin-A levels [43]. Overweight women with T2DM were instructed by a dietitian on how to reduce energy intake by $30 \%$ (an average of $586 \mathrm{kcal}$ per day) over a period of 12 weeks. During this time, Fetuin-A decreased by $7 \%$ when compared to a healthy control group $(0.23 \mathrm{mg} / \mathrm{mL}$ to $0.25 \mathrm{mg} / \mathrm{mL})$. Herein circulating free fatty acids were not significantly different after this calorie restriction intervention [43]. Conversely, Hwang and colleagues found that calorie restriction - short or long-term - had no impact on Fetuin-A [86]. Importantly, the calorie restriction in this study was not designed as an intervention, rather the study participants were defined as calorically restricted due to intentional, excessive exercise and 
low body weight. This report also found no differences in short-term calorie restriction (72 hour fasting) in men and women with a BMI less than $25 \mathrm{~kg} / \mathrm{m}^{2}$ [86]. Therefore, the impact of calorie restriction on circulating Fetuin-A may depend on baseline Fetuin-A levels and body composition or adipose tissue distribution.

Due to the proposed role of Fetuin-A in insulin resistance, it has been studied in relation to specific foods associated with T2DM development or management. Examples include n-3 PUFA, coffee and alcohol [87-90]. Since increased PUFA intake has been associated with improved insulin sensitivity [91], an intervention was designed to supplement n-3 PUFA (1.2 g/day) to 40 diabetic patients $[89,91]$. Ozyazgan and collaborators found that after two months of PUFA supplementation, serum Fetuin-A levels were decreased [89]. This study also found positive baseline associations between serum Fetuin-A and triglycerides and HbAlc levels, but it's not clear if the dietary supplementation modified the correlations of circulating Fetuin-A and the lipid profile [89]. Still, there are no studies reporting on the effects of PUFA supplementation on Fetuin$\mathrm{A}$ in individuals without T2DM.

Epidemiological evidence suggests that coffee drinkers have a lower risk for T2DM [92]. To determine whether Fetuin-A was influenced by coffee consumption, overweight individuals who drank five cups of caffeinated or decaffeinated coffee daily for 8 weeks were compared to overweight individuals who drank five cups of water daily for 8 weeks. After this intervention, those who drank decaffeinated coffee daily reduced Fetuin-A by $20 \%$ and had significantly lower Fetuin-A than those who did not drink any coffee. Consumption of caffeinated coffee reduced Fetuin-A by $11 \%$, but this effect was not statistically significant [90].

Alcohol intake in moderation has been associated with a lower risk of T2DM development. Accordingly, alcohol consumption was negatively correlated to Fetuin-A in the Nurses' Health 
Study [88]. Importantly, this consumption data was self-reported and retrospective. When this finding was investigated in a randomized crossover trial, it was found that the type of alcoholic beverage may be important. Consumption of white wine ( $25 \mathrm{~g}$ alcohol) versus grape juice daily for 6 weeks did not modify the Fetuin-A levels of 36 postmenopausal women [87]. Still, in a crossover study, 24 men who consumed 100mL of vodka daily for 4 weeks had significantly lower Fetuin-A than when they consumed only orange juice daily for 4 weeks. However the decrease in Fetuin-A was minute $(0.44$ and $0.43 \mathrm{mg} / \mathrm{ml}$, respectively) [87]. The molecular or physiological mechanisms for this finding are yet to be elucidated.

\subsection{Exercise}

Only a handful of studies have attempted to evaluate the impact of a physical activity intervention on Fetuin-A [45, 93-95]. Seven days of aerobic exercise training resulted in an 11\% decrease in Fetuin-A without significant weight loss in 12 obese adults [93]. In order to replicate their findings, these same researchers administered a 12-week exercise program to 20 older adults and found that an average weight loss of $6.4 \mathrm{~kg}$ led to an $8 \%$ reduction in Fetuin-A [45]. Conversely, a different group of researchers reported no impact on Fetuin-A following a 6-week intervention consisted of aerobic exercise three times per week. This intervention included 14 obese and non-diabetic women and even though no significant changes in Fetuin-A occurred, significant reductions in body fat and waist circumference were seen [95]. Mathews and colleagues administered four bouts of daily exercise (designed to expend 350 calories each) and also found no significant changes in total Fetuin-A concentration [94]. However, this exercise did lead to decreased phosphorylation of Fetuin-A at Ser312 twenty-four hours after intervention. To our 
knowledge, this is the only report that underlines the effect of exercise on post-translational modifications of Fetuin-A [94].

It is possible that exercise style, duration and intensity play a role on Fetuin-A and phosphofetuin levels. However, given the scarcity of data, at this time, it is not possible to conclude what the impact of exercise is on circulating Fetuin-A. Future studies should describe in detail the type and duration of exercise, and measure differences in post-translational modification of circulating Fetuin-A.

\subsection{Aging}

Fetuin-A may also be impacted by the natural aging process. Much of our understanding of Fetuin-A's role in older adults comes from two cohorts, ABC Health and the Rancho Bernardo Study [5, 23, 79]. The Rancho Bernardo study included participants with a median age of 71 years and found that Fetuin-A decreases with age. Women taking estrogen had the highest Fetuin-A followed by women not taking estrogen. Men had the lowest Fetuin-A levels. Another important sex difference that was pointed out in this study was the association between Fetuin-A and T2DM risk in women but not in men $[7,96,97]$. In contrast to the Ranch Bernardo Study, the EPICPotsdam study found that plasma Fetuin-A levels predicted increased risk for incident T2DM in men and women (35 to 65 year-old) during an average of 7 years of follow-up [74, 98].

The process of aging typically results in decreased insulin sensitivity, however, Fetuin-A knock out mice appeared to be protected against age-associated insulin resistance at 80 weeks (approximately 53 human years) [99]. Epidemiological evidence suggests that older adults with the lowest Fetuin-A are at a decreased risk for T2DM. The Nurses' Health Study found that women in the highest quintile (Fetuin-A $\geq 0.57 \mathrm{mg} / \mathrm{mL}$ ) had around a 1.8 fold higher risk of T2DM than 
women at the lowest quintile (Fetuin-A $\leq 0.40 \mathrm{mg} / \mathrm{mL}$ ) [63]. Similarly, the Health ABC study found that older individuals with the highest tertile of Fetuin-A $(>0.97 \mathrm{mg} / \mathrm{mL})$ had a two-fold higher incident rate of T2DM than individuals with the lowest tertile of Fetuin-A $(\leq 0.76 \mathrm{mg} / \mathrm{mL})$ [5]. Likewise, the EPIC-Potsdam study found that risk for incident T2DM increased 1.75 times (95\% CI: 1.32-2.31) after adjustment for age in extreme quintiles of Fetuin-A [98].

In regards to aging, Fetuin-A has been suggested to be a marker of cognitive decline [7]. In a study of 1,382 older individuals, those with higher Fetuin-A performed better than those with lower Fetuin-A on a series of tasks designed to assess attention, calculation, orientation, language and recall. Those with higher Fetuin-A were also less likely to experience cognitive declines longitudinally [7]. In an earlier study of cognitive impairment, it was found that plasma Fetuin-A was significantly lower in of 34 individuals with Alzheimer's disease (AD) than in 34 age-matched controls $(0.26 \mathrm{mg} / \mathrm{mL}$ and $0.30 \mathrm{mg} / \mathrm{mL}$, respectively) [100]. Genetics may also influence the association of Fetuin-A with AD. A study of 235 Italians found that late-onset AD was 3.9 times more likely if the individual had a haplotype of two SNPs in the AHSG gene (rs4917 and rs4918) $[100,101]$. Although the sample size of many of the studies revised is small, they represent an emerging area of research in the study of this versatile protein. At this time, it is unclear how Fetuin-A contributes to cognition but these findings suggest that higher Fetuin-A may serve an important role in preserving the cognitive abilities of older adults.

\section{Conclusion: Challenges to studying Fetuin-A}

The objective of this review was to discuss factors that influence Fetuin-A throughout the lifecycle including modifiable life factors such as diet, exercise, weight management and elective surgeries. This literature review revealed a set of challenges to the study of Fetuin-A in humans 
and its relevance as a metabolic biomarker (Table 1). First, reference values and standard units of measure have not yet been established. In the study of older adults, it is common for the analytical sample to be split into tertiles or quintiles for analysis. In doing so, the ability to create a standard reference of circulating Fetuin-A is lessened as it becomes specific to each cohort studied and only related to the outcome variable being assessed. Secondly, comparisons across studies are difficult due to variability in commercially available kits. This may be because the kits are sensitive to posttranslational modifications such as glycosylation [53]. Measuring post-translationally modified forms of Fetuin-A (i.e. glycosylated or phosphorylated) may be more informative in the study of chronic disease than total circulating Fetuin-A $[11,15]$.

Few studies have examined the temporal variation in Fetuin-A within individuals. FetuinA appears to vary week-to-week, but it is not clear if this relationship is related to sexual dimorphism [102]. At the time of this review, no study evaluating Fetuin-A fluctuations throughout the day has been reported. However, Fetuin-A does appear to vary throughout the day based on meal intake [58]. Thus, as this field moves forward, it is important for researchers to disclose whether measurements are collected while fasting and for how many hours the participants have been fasting. Due to the multi-functionality of Fetuin-A, it can also be challenging to identify an appropriate clinical control group for comparison. Ideally, control group individuals should be age-, sex- and weight-matched to the treatment or intervention group. Attention to renal and liver function, insulin sensitivity levels, acute infection, medications and genetic factors should be noted. Furthermore, our search revealed that physical activity and nutrition are important influences and should be described in detail when evaluating Fetuin-A in human participants.

\section{Insert Table 1 about here}




\section{Summary}

Fetuin-A is a multifunctional protein that inhibits ectopic calcification and insulin signaling via tyrosine kinase. Increased levels of Fetuin-A have been linked to greater risk of CVD and incident T2DM, therefore, it has been proposed as a biomarker for these diseases. In order to fully understand the clinical utility of Fetuin-A, consideration must be given to the multiple factors influencing Fetuin-A beyond the presence of these diseases. Fetuin-A is affected by multiple biological and behavioral factors. Biological factors include genetics and aging among others. Behavioral factors that impact Fetuin-A levels include physical activity, weight loss and dietary intake.

Throughout the lifecycle, Fetuin A is highest at infancy (around $1 \mathrm{mg} / \mathrm{mL}$ ) and remains stable throughout childhood and adulthood but values vary. At this time, it is unclear how FetuinA during infancy is impacted by early life exposure including maternal diet, maternal weight status and breastfeeding. Fetuin-A research would also benefit from studies on specific age ranges and important developmental periods. Individual genetic variation is another biological factor which influences Fetuin-A in circulation and if not considered, may skew findings and preclude advancing the field of Fetuin-A research.

Behavioral factors can also influence Fetuin-A. In general, physical activity appears to decrease Fetuin-A and weight loss either by lifestyle change or weight loss surgery, also decreases Fetuin-A. Although research concerning the dietary impact on Fetuin-A is sparse, it appears that excess calorie intake increases Fetuin-A while low-calorie diets reduce Fetuin-A. Therefore, if Fetuin-A does prove to be an informative clinical marker of CVD or T2DM, it may be possible to modify circulating Fetuin-A through physical activity, weight management and nutritional interventions. 
Several disciplines such as biology, medicine, genetics, kinesiology and nutrition have contributed to the overall understanding of Fetuin-A in recent years. Yet, this enigmatic protein has eluded consensus on reference values and a true understanding of its biological importance. Therefore, Fetuin-A research may provide opportunities for transdisciplinary collaborations between benchtop scientists and clinicians to determine ideal Fetuin-A values and whether nonpharmacological management strategies are effective at altering Fetuin-A and thus reducing disease progression.

\section{Acknowledgements}

Katie Robinson is supported by the National Institute for Agriculture U.S. Department of Agriculture, under award number 2011-67001-30101 for the Illinois Transdisciplinary Obesity Prevention Program to the Division of Nutritional Sciences at the University of Illinois. Margarita Teran-Garcia is supported by the National Institute for Agriculture under award number ILLU689-312. Any opinions, findings, conclusions, or recommendations expressed in this publication are those of the author(s) and do not necessarily reflect the view of the U.S. Department of Agriculture. 


\section{References}

[1] K.O. Pedersen, Fetuin, a new globulin isolated from serum, Nature, 154 (1944) 575.

[2] P.R. Srinivas, A.S. Wagner, L.V. Reddy, D.D. Deutsch, M.A. Leon, A.S. Goustin, G. Grunberger, Serum alpha 2-HS-glycoprotein is an inhibitor of the human insulin receptor at the tyrosine kinase level, Mol. Endocrinol., 7 (1993) 1445-1455.

[3] P. Arnaud, L. Kalabay, Alpha2-HS glycoprotein: a protein in search of a function, Diabetes Metab. Res. Rev., 18 (2002) 311-314.

[4] N. Stefan, A.M. Hennige, H. Staiger, J. Machann, F. Schick, S.M. Krober, F. Machicao, A. Fritsche, H.U. Häring, Alpha2-Heremans-Schmid glycoprotein/fetuin-A is associated with insulin resistance and fat accumulation in the liver in humans, Diabetes Care, 29 (2006) 853-857.

[5] J.H. Ix, C.L. Wassel, A.M. Kanaya, et al., Fetuin-A and incident diabetes mellitus in older persons, JAMA, 300 (2008) 182-188.

[6] N. Stefan, H.U. Häring, Circulating fetuin-A and free fatty acids interact to predict insulin resistance in humans, Nat. Med., 19 (2013) 394-395.

[7] G.A. Laughlin, L.K. McEvoy, E. Barrett-Connor, L.B. Daniels, J.H. Ix, Fetuin-A, a new vascular biomarker of cognitive decline in older adults, Clin. Endocrinol., 81 (2014) 134-140.

[8] K.M. Dziegielewska, K. Møllgård, M.L. Reynolds, N.R. Saunders, A fetuin-related glycoprotein (alpha 2HS) in human embryonic and fetal development, Cell Tissue Res., 248 (1987) $33-41$.

[9] B. Denecke, S. Graber, C. Schafer, A. Heiss, M. Woltje, W. Jahnen-Dechent, Tissue distribution and activity testing suggest a similar but not identical function of fetuin-B and fetuinA, Biochem. J., 376 (2003) 135-145. 
[10] K.M. Dziegielewska, W.M. Brown, Fetuin, Springer-Verlag Berlin Heidelberg, 1995, pp. VIII, 180.

[11] A.S. Goustin, A.B. Abou-Samra, The "thrifty" gene encoding Ahsg/Fetuin-A meets the insulin receptor: Insights into the mechanism of insulin resistance, Cell. Signal., 23 (2011) 980990.

[12] A.S. Goustin, N. Derar, A.B. Abou-Samra, AHSG-fetuin blocks the metabolic arm of insulin action through its interaction with the $95-\mathrm{kD}$ beta-subunit of the insulin receptor, Cell. Signal., 25 (2013) 981-988.

[13] C.C. Lee, B.H. Bowman, F.M. Yang, Human alpha 2-HS-glycoprotein: the A and B chains with a connecting sequence are encoded by a single mRNA transcript, Proc. Natl. Acad. Sci. U.S.A., 84 (1987) 4403-4407.

[14] Y. Yoshioka, F. Gejyo, T. Marti, E.E. Rickli, W. Burgi, G.D. Offner, R.F. Troxler, K. Schmid, The complete amino acid sequence of the A-chain of human plasma alpha 2HS-glycoprotein, J. Biol. Chem., 261 (1986) 1665-1676.

[15] A.C. Haglund, B. Ek, P. Ek, Phosphorylation of human plasma alpha2-Heremans-Schmid glycoprotein (human fetuin) in vivo, Biochem. J., 357 (2001) 437-445.

[16] P. Auberger, L. Falquerho, J.O. Contreres, G. Pages, G. Le Cam, B. Rossi, A. Le Cam, Characterization of a natural inhibitor of the insulin receptor tyrosine kinase: cDNA cloning, purification, and anti-mitogenic activity, Cell, 58 (1989) 631-640.

[17] M. Demetriou, C. Binkert, B. Sukhu, H.C. Tenenbaum, J.W. Dennis, Fetuin/alpha2-HS glycoprotein is a transforming growth factor-beta type II receptor mimic and cytokine antagonist, J. Biol. Chem., 271 (1996) 12755-12761. 
[18] W. Jahnen-Dechent, A. Heiss, C. Schäfer, M. Ketteler, Fetuin-A Regulation of Calcified Matrix Metabolism, Circ. Res., 108 (2011) 1494-1509.

[19] A. Heiss, V. Pipich, W. Jahnen-Dechent, D. Schwahn, Fetuin-A is a mineral carrier protein: small angle neutron scattering provides new insight on Fetuin-A controlled calcification inhibition, Biophys. J., 99 (2010) 3986-3995.

[20] M.M. Cai, E.R. Smith, S.G. Holt, The role of fetuin-A in mineral trafficking and deposition, BoneKEy Rep., 4 (2015) 672.

[21] L. Brylka, W. Jahnen-Dechent, The role of fetuin-A in physiological and pathological mineralization, Calcif. Tissue Int., 93 (2013) 355-364.

[22] M. Herrmann, C. Schafer, A. Heiss, S. Graber, A. Kinkeldey, A. Buscher, M.M. Schmitt, J. Bornemann, F. Nimmerjahn, L. Helming, S. Gordon, W. Jahnen-Dechent, Clearance of fetuin-A-containing calciprotein particles is mediated by scavenger receptor-A, Circ. Res., 111 (2012) 575584.

[23] M. Ketteler, P. Bongartz, R. Westenfeld, J. Wildberger, A. Mahnken, R. Bohm, T. Metzger, C. Wanner, W. Jahnen-Dechent, J. Floege, Association of low fetuin-A (AHSG) concentrations in serum with cardiovascular mortality in patients on dialysis: a cross-sectional study, Lancet, 361 (2003) 827-833.

[24] C. Weikert, N. Stefan, M.B. Schulze, T. Pischon, K. Berger, H.G. Joost, H.U. Haring, H. Boeing, A. Fritsche, Plasma fetuin-a levels and the risk of myocardial infarction and ischemic stroke, Circulation, 118 (2008) 2555-2562.

[25] Q. Sun, M.C. Cornelis, J.E. Manson, F.B. Hu, Plasma levels of fetuin-A and hepatic enzymes and risk of type 2 diabetes in women in the U.S., Diabetes, 62 (2013) 49-55. 
[26] L. Yin, W.J. Cai, L.Y. Zhu, J. Li, X.H. Su, X.L. Wang, X.Y. Chang, K. Sun, Association of plasma Fetuin-A and clinical characteristics in patients with new-onset type 2 diabetes mellitus, Int. J. Clin. Exp. Med., 8 (2015) 991-999.

[27] S.T. Mathews, P.R. Srinivas, M.A. Leon, G. Grunberger, Bovine fetuin is an inhibitor of insulin receptor tyrosine kinase, Life Sci., 61 (1997) 1583-1592.

[28] S.T. Mathews, N. Chellam, P.R. Srinivas, V.J. Cintron, M.A. Leon, A.S. Goustin, G. Grunberger, Alpha2-HSG, a specific inhibitor of insulin receptor autophosphorylation, interacts with the insulin receptor, Mol. Cell. Endocrinol., 164 (2000) 87-98.

[29] D. Pal, S. Dasgupta, R. Kundu, S. Maitra, G. Das, S. Mukhopadhyay, S. Ray, S.S. Majumdar, S. Bhattacharya, Fetuin-A acts as an endogenous ligand of TLR4 to promote lipid-induced insulin resistance, Nat. Med., 18 (2012) 1279-1285.

[30] P. Chatterjee, S. Seal, S. Mukherjee, R. Kundu, S. Ray, S. Mukhopadhyay, S.S. Majumdar, S. Bhattacharya, Adipocyte fetuin-A contributes to macrophage migration into adipose tissue and polarization of macrophages, J. Biol. Chem., 288 (2013) 28324-28330.

[31] C. Akhoundi, M. Amiot, P. Auberger, A. Le Cam, B. Rossi, Insulin and interleukin-1 differentially regulate pp63, an acute phase phosphoprotein in hepatoma cell line, J. Biol. Chem., 269 (1994) 15925-15930.

[32] K.M. Dziegielewska, W.M. Brown, C.C. Gould, N. Matthews, J.E. Sedgwick, N.R. Saunders, Fetuin: an acute phase protein in cattle, J. Comp. Physiol. B., 162 (1992) 168-171.

[33] J.P. Lebreton, F. Joisel, J.P. Raoult, B. Lannuzel, J.P. Rogez, G. Humbert, Serum concentration of human alpha 2 HS glycoprotein during the inflammatory process: evidence that alpha 2 HS glycoprotein is a negative acute-phase reactant, J. Clin. Invest., 64 (1979) 1118-1129. 
[34] W. Li, S. Zhu, J. Li, Y. Huang, R. Zhou, X. Fan, H. Yang, X. Gong, N.T. Eissa, W. JahnenDechent, P. Wang, K.J. Tracey, A.E. Sama, H. Wang, A hepatic protein, fetuin-A, occupies a protective role in lethal systemic inflammation, PLoS One, 6 (2011) 0016945.

[35] K.E. Sandoval, K.A. Witt, Blood-brain barrier tight junction permeability and ischemic stroke, Neurobiol. Dis., 32 (2008) 200-219.

[36] H. Wang, A.E. Sama, Anti-inflammatory role of fetuin-A in injury and infection, Curr. Mol. Med., 12 (2012) 625-633.

[37] H. Wang, M. Zhang, K. Soda, A. Sama, K.J. Tracey, Fetuin protects the fetus from TNF, Lancet, 350 (1997) 861-862.

[38] P. Zhang, H. Shen, J. Huang, H. Wang, B. Zhang, R. Zhou, B. Zhong, X. Fan, Intraperitoneal administration of Fetuin-A attenuates D-galactosamine/lipopolysaccharide-induced liver failure in mouse, Dig. Dis. Sci., 59 (2014) 1789-1797.

[39] K.M. Dziegielewska, N. Matthews, N.R. Saunders, G. Wilkinson, alpha 2HS-glycoprotein is expressed at high concentration in human fetal plasma and cerebrospinal fluid, Fetal Diagn. Ther., 8 (1993) 22-27.

[40] J. Seto, B. Busse, H.S. Gupta, C. Schafer, S. Krauss, J.W. Dunlop, A. Masic, M. Kerschnitzki, P. Zaslansky, P. Boesecke, P. Catala-Lehnen, T. Schinke, P. Fratzl, W. Jahnen-Dechent, Accelerated growth plate mineralization and foreshortened proximal limb bones in fetuin-A knockout mice, PLoS One, 7 (2012) e47338.

[41] M. Häusler, C. Schäfer, C. Osterwinter, W. Jahnen-Dechent, The Physiologic Development of Fetuin-A Serum Concentrations in Children, Pediatr. Res., 66 (2009) 660-664.

[42] M. Wigger, J. Schaible, J. Muscheites, G. Kundt, D. Haffner, D.C. Fischer, Fetuin-A serum concentrations in healthy children, Ann. Clin. Biochem., 46 (2009) 511-513. 
[43] K.M. Choi, K.A. Han, H.J. Ahn, S.Y. Lee, S.Y. Hwang, B.H. Kim, H.C. Hong, H.Y. Choi, S.J. Yang, H.J. Yoo, S.H. Baik, D.S. Choi, K.W. Min, The effects of caloric restriction on fetuinA and cardiovascular risk factors in rats and humans: a randomized controlled trial, Clin. Endocrinol., 79 (2013) 356-363.

[44] J.M. Brix, H. Stingl, F. Hollerl, G.H. Schernthaner, H.P. Kopp, G. Schernthaner, Elevated Fetuin-A concentrations in morbid obesity decrease after dramatic weight loss, J. Clin. Endocrinol. Metab., 95 (2010) 4877-4881.

[45] S.K. Malin, J.P. del Rincon, H. Huang, J.P. Kirwan, Exercise-induced lowering of fetuin-A may increase hepatic insulin sensitivity, Med. Sci. Sports Exerc., 46 (2014) 2085-2090.

[46] D. Samocha-Bonet, C.S. Tam, L.V. Campbell, L.K. Heilbronn, Raised circulating Fetuin-A after 28-day overfeeding in healthy humans, Diabetes Care, 37 (2014) E15-E16.

[47] G. Rauth, O. Poschke, E. Fink, M. Eulitz, S. Tippmer, M. Kellerer, H.U. Haring, P. Nawratil, M. Haasemann, W. Jahnen-Dechent, et al., The nucleotide and partial amino acid sequences of rat fetuin. Identity with the natural tyrosine kinase inhibitor of the rat insulin receptor, Eur. J. Biochem., 204 (1992) 523-529.

[48] J.F. Trepanowski, J. Mey, K.A. Varady, Fetuin-A: a novel link between obesity and related complications, Int. J. Obes., 39 (2015) 734-741.

[49] K. Vörös, L.J. Gráf, Z. Prohászka, L. Gráf, P. Szenthe, E. Kaszás, Z. Böröcz, K. Cseh, L. Kalabay, Serum fetuin-A in metabolic and inflammatory pathways in patients with myocardial infarction, Eur. J. Clin. Invest., 41 (2011) 703-709.

[50] D.C. Fischer, J. Schaible, M. Wigger, H. Staude, E. Drueckler, G. Kundt, D. Haffner, Reduced serum fetuin-A in nephrotic children: a consequence of proteinuria?, Am. J. Nephrol., 34 (2011) 373-380. 
[51] D.M. Lebensztejn, I. Bialokoz-Kalinowska, M. Klusek-Oksiuta, E. Tarasów, M. Wojtkowska, M. Kaczmarski, Serum fetuin A concentration is elevated in children with non-alcoholic fatty liver disease, Adv. Med. Sci., 59 (2014) 81-84.

[52] T. Reinehr, C.L. Roth, Fetuin-A and its relation to metabolic syndrome and fatty liver disease in obese children before and after weight loss, J. Clin. Endocrinol. Metab., 93 (2008) 4479-4485. [53] E.R. Smith, M.L. Ford, L.A. Tomlinson, B.F. Rocks, C. Rajkumar, S.G. Holt, Poor agreement between commercial ELISAs for plasma fetuin-A: An effect of protein glycosylation?, Clin. Chim. Acta, 411 (2010) 1367-1370.

[54] A. Heiss, T. Eckert, A. Aretz, W. Richtering, W. van Dorp, C. Schafer, W. Jahnen-Dechent, Hierarchical role of fetuin-A and acidic serum proteins in the formation and stabilization of calcium phosphate particles, J. Biol. Chem., 283 (2008) 14815-14825.

[55] C. Binkert, M. Demetriou, B. Sukhu, M. Szweras, H.C. Tenenbaum, J.W. Dennis, Regulation of osteogenesis by fetuin, J. Biol. Chem., 274 (1999) 28514-28520.

[56] Z. Nie, Fetuin: its enigmatic property of growth promotion, Am. J. Physiol., 263 (1992) C551562.

[57] L. Kalabay, K. Cseh, A. Pajor, E. Baranyi, G.M. Csakany, Z. Melczer, G. Speer, M. Kovacs, G. Siller, I. Karádi, G. Winkler, Correlation of maternal serum fetuin/alpha(2)-HS-glycoprotein concentration with maternal insulin resistance and anthropometric parameters of neonates in normal pregnancy and gestational diabetes, Eur. J. Endocrinol., 147 (2002) 243-248.

[58] S. Farhan, A. Handisurya, J. Todoric, A. Tura, G. Pacini, O. Wagner, K. Klein, R. Jarai, K. Huber, A. Kautzky-Willer, Fetuin-A Characteristics during and after Pregnancy: Result from a Case Control Pilot Study, Int. J. Endocrinol., 2012 (2012) ID: 896736. 
[59] L.M. Gómez, Understanding the effects of fetuin-A in pregnancy, Med. J. Obstet. Gynecol., $1(2013) 1010$.

[60] Y. Dautova, D. Kozlova, J.N. Skepper, M. Epple, M.D. Bootman, D. Proudfoot, Fetuin-A and albumin alter cytotoxic effects of calcium phosphate nanoparticles on human vascular smooth muscle cells, PLoS One, 9 (2014) e97565.

[61] D.D. Briana, M. Boutsikou, D. Gourgiotis, T. Boutsikou, S. Baka, A. Marmarinos, D. Hassiakos, A. Malamitsi-Puchner, Serum fetuin-A/alpha2-HS-glycoprotein in human pregnancies with normal and restricted fetal growth, J. Matern. Fetal Neonatal Med., 21 (2008) 826-830.

[62] P.M. Karamessinis, A. Malamitsi-Puchner, T. Boutsikou, M. Makridakis, K. Vougas, M. Fountoulakis, A. Vlahou, G. Chrousos, Marked defects in the expression and glycosylation of alpha(2)-HS glycoprotein/fetuin-A in plasma from neonates with intrauterine growth restriction Proteomics screening and potential clinical implications, Mol. Cell. Proteomics, 7 (2008) 591-599. [63] N. Stefan, H.U. Häring, F.B. Hu, M.B. Schulze, Metabolically healthy obesity: epidemiology, mechanisms, and clinical implications, Lancet Diabetes Endocrinol., 1 (2013) 152-162.

[64] J.P. Rey-López, L.F. de Rezende, T.H. de Sá, E. Stamatakis, Is the Metabolically Healthy Obesity Phenotype an Irrelevant Artifact for Public Health?, Am. J. Epidemiol., (2015).

[65] J.V. van Vliet-Ostaptchouk, M.L. Nuotio, S.N. Slagter, D. Doiron, K. Fischer, L. Foco, A. Gaye, M. Gogele, M. Heier, T. Hiekkalinna, A. Joensuu, C. Newby, C. Pang, E. Partinen, E. Reischl, C. Schwienbacher, M.L. Tammesoo, M.A. Swertz, P. Burton, V. Ferretti, I. Fortier, L. Giepmans, J.R. Harris, H.L. Hillege, J. Holmen, A. Jula, J.E. Kootstra-Ros, K. Kvaloy, T.L. Holmen, S. Mannisto, A. Metspalu, K. Midthjell, M.J. Murtagh, A. Peters, P.P. Pramstaller, T. Saaristo, V. Salomaa, R.P. Stolk, M. Uusitupa, P. van der Harst, M.M. van der Klauw, M. Waldenberger, M. Perola, B.H. Wolffenbuttel, The prevalence of metabolic syndrome and 
metabolically healthy obesity in Europe: a collaborative analysis of ten large cohort studies, BMC Endocr. Disord., 14 (2014) 9.

[66] C.K. Kramer, B. Zinman, R. Retnakaran, Are metabolically healthy overweight and obesity benign conditions?: A systematic review and meta-analysis, Ann. Intern. Med., 159 (2013) 758769.

[67] N. Stefan, H.U. Häring, The role of hepatokines in metabolism, Nat. Rev. Endocrinol., 9 (2013) 144-152.

[68] N. Stefan, H.U. Häring, The metabolically benign and malignant fatty liver, Diabetes, 60 (2011) 2011-2017.

[69] C.E. Elks, M. den Hoed, J.H. Zhao, S.J. Sharp, N.J. Wareham, R.J. Loos, K.K. Ong, Variability in the heritability of body mass index: a systematic review and meta-regression, Front. Endocrinol., 3 (2012) 29.

[70] C. Lavebratt, S. Wahlqvist, L. Nordfors, J. Hoffstedt, P. Arner, AHSG gene variant is associated with leanness among Swedish men, Hum. Genet., 117 (2005) 54-60.

[71] G. Temesszentandrasi, K. Vörös, Z. Borocz, E. Kaszás, Z. Prohászka, A. Falus, K. Cseh, L. Kalabay, Association of Human Fetuin-A rs4917 Polymorphism With Obesity in 2 Cohorts, J. Investig. Med., 63 (2015) 548-553.

[72] N. Vionnet, E.H. Hani, S. Dupont, S. Gallina, S. Francke, S. Dotte, F. De Matos, E. Durand, F. Lepretre, C. Lecoeur, P. Gallina, L. Zekiri, C. Dina, P. Froguel, Genomewide search for type 2 diabetes-susceptibility genes in French whites: evidence for a novel susceptibility locus for earlyonset diabetes on chromosome 3q27-qter and independent replication of a type 2-diabetes locus on chromosome 1q21-q24, Am. J. Hum. Genet., 67 (2000) 1470-1480. 
[73] A.C. Choquette, S. Lemieux, A. Tremblay, Y.C. Chagnon, C. Bouchard, M.C. Vohl, L. Perusse, Evidence of a quantitative trait locus for energy and macronutrient intakes on chromosome 3q27.3: the Quebec Family Study, Am. J. Clin. Nutr., 88 (2008) 1142-1148.

[74] E. Fisher, N. Stefan, K. Saar, D. Drogan, M.B. Schulze, A. Fritsche, H.G. Joost, H.U. Haring, N. Hubner, H. Boeing, C. Weikert, Association of AHSG gene polymorphisms with fetuin-A plasma levels and cardiovascular diseases in the EPIC-Potsdam study, Circ. Cardiovasc. Genet., 2 (2009) 607-613.

[75] M.K. Jensen, T.M. Bartz, L. Djousse, J.R. Kizer, S.J. Zieman, E.B. Rimm, D.S. Siscovick, B.M. Psaty, J.H. Ix, K.J. Mukamal, Genetically elevated fetuin-A levels, fasting glucose levels, and risk of type 2 diabetes: the cardiovascular health study, Diabetes Care, 36 (2013) 3121-3127. [76] I. Dahlman, P. Eriksson, M. Kaaman, H. Jiao, C.M. Lindgren, J. Kere, P. Arner, alpha2Heremans-Schmid glycoprotein gene polymorphisms are associated with adipocyte insulin action, Diabetologia, 47 (2004) 1974-1979.

[77] K. Müssig, H. Staiger, F. Machicao, J. Machann, A.M. Hennige, F. Schick, C.D. Claussen, A. Fritsche, H.U. Häring, N. Stefan, AHSG gene variation is not associated with regional body fat distribution--a magnetic resonance study, Exp. Clin. Endocrinol. Diabetes, 117 (2009) 432-437.

[78] M. Jüllig, S. Yip, A. Xu, G. Smith, M. Middleditch, M. Booth, R. Babor, G. Beban, R. Murphy, Lower Fetuin-A, Retinol Binding Protein 4 and Several Metabolites after Gastric Bypass Compared to Sleeve Gastrectomy in Patients with Type 2 Diabetes, PLoS One, 9 (2014) e96489. [79] J.H. Ix, C.L. Wassel, G.M. Chertow, A. Koster, K.C. Johnson, F.A. Tylavsky, J.A. Cauley, S.R. Cummings, T.B. Harris, M.G. Shlipak, Fetuin-A and Change in Body Composition in Older Persons, J. Clin. Endocrinol. Metab. , 94 (2009) 4492-4498. 
[80] A. Ochi, K. Mori, M. Emoto, S. Nakatani, T. Morioka, K. Motoyama, S. Fukumoto, Y. Imanishi, H. Koyama, E. Ishimura, M. Inaba, Direct inhibitory effects of pioglitazone on hepatic fetuin-A expression, PLoS One, 9 (2014) e88704.

[81] J.W. Haukeland, T.B. Dahl, A. Yndestad, I.P. Gladhaug, E.M. Loberg, T. Haaland, Z. Konopski, C. Wium, E.T. Aasheim, O.E. Johansen, P. Aukrust, B. Halvorsen, K.I. Birkeland, Fetuin A in nonalcoholic fatty liver disease: in vivo and in vitro studies, Eur. J. Endocrinol., 166 (2012) 503-510.

[82] K. Mori, M. Emoto, T. Araki, H. Yokoyama, E. Lee, M. Teramura, H. Koyama, T. Shoji, M. Inaba, Y. Nishizawa, Effects of pioglitazone on serum fetuin-A levels in patients with type 2 diabetes mellitus, Metabolism, 57 (2008) 1248-1252.

[83] S.V. Kaushik, E.P. Plaisance, T. Kim, E.Y. Huang, A.J. Mahurin, P.W. Grandjean, S.T. Mathews, Extended-release niacin decreases serum fetuin-A concentrations in individuals with metabolic syndrome, Diabetes Metab. Res. Rev, 25 (2009) 427-434.

[84] M. Woltje, B. Tschoke, V. von Bulow, R. Westenfeld, B. Denecke, S. Graber, W. JahnenDechent, CCAAT enhancer binding protein beta and hepatocyte nuclear factor 3beta are necessary and sufficient to mediate dexamethasone-induced up-regulation of alpha2HS-glycoprotein/fetuinA gene expression, J. Mol. Endocrinol., 36 (2006) 261-277.

[85] K. Nimptsch, J. Janke, T. Pischon, J. Linseisen, Association between dietary factors and plasma fetuin-A concentrations in the general population, Br. J. Nutr., 114 (2015) 1278-1285.

[86] J.J. Hwang, B. Thakkar, J.P. Chamberland, C.S. Mantzoros, Circulating fetuin-A levels are not affected by short and long-term energy deprivation and/or by leptin administration, Metabolism, 63 (2014) 754-759. 
[87] M.M. Joosten, I.C. Schrieks, H.F. Hendriks, Effect of moderate alcohol consumption on fetuin-A levels in men and women: post-hoc analyses of three open-label randomized crossover trials, Diabetol. Metab. Syndr., 6 (2014) 24.

[88] S.H. Ley, Q. Sun, M.C. Jimenez, K.M. Rexrode, J.E. Manson, M.K. Jensen, E.B. Rimm, F.B. $\mathrm{Hu}$, Association between alcohol consumption and plasma fetuin-A and its contribution to incident type 2 diabetes in women, Diabetologia, 57 (2014) 93-101.

[89] S. Ozyazgan, K. Karaoglu, A. Kurt, A. Altinok, D. Konukoglu, Z. Osar Siva, G. Andican, Effects of omega-3 polyunsaturated fatty acid supplementation on serum fetuin-A levels in type 2 diabetic patients, Minerva Med., 104 (2013) 287-293.

[90] N.M. Wedick, A.M. Brennan, Q. Sun, F.B. Hu, C.S. Mantzoros, R.M. van Dam, Effects of caffeinated and decaffeinated coffee on biological risk factors for type 2 diabetes: a randomized controlled trial, Nutr. J., 10 (2011) 93.

[91] L.K. Summers, B.A. Fielding, H.A. Bradshaw, V. Ilic, C. Beysen, M.L. Clark, N.R. Moore, K.N. Frayn, Substituting dietary saturated fat with polyunsaturated fat changes abdominal fat distribution and improves insulin sensitivity, Diabetologia, 45 (2002) 369-377.

[92] R.M. Van Dam, F.B. Hu, Coffee consumption and risk of type 2 diabetes: a systematic review, JAMA, 294 (2005) 97-104.

[93] S.K. Malin, A. Mulya, C.E. Fealy, J.M. Haus, M.R. Pagadala, A.R. Scelsi, H. Huang, C.A. Flask, A.J. McCullough, J.P. Kirwan, Fetuin-A is linked to improved glucose tolerance after shortterm exercise training in nonalcoholic fatty liver disease, J. Appl. Physiol., 115 (2013) 988-994.

[94] S. Mathews, G. Ren, X. He, R. Bowers, F. Araya-Ramirez, L. Littlefield, P. Grandjean, Plasma fetuin-A and phosphofetuin-A (Ser312) responses to a single or short-term repeated bout 
of exercise in obese and normal-weight individuals (1028.2), The FASEB Journal, 28 (2014) 1028.1022.

[95] B. Schultes, J. Frick, B. Ernst, N. Stefan, A. Fritsche, The effect of 6-weeks of aerobic exercise training on serum fetuin-A levels in non-diabetic obese women, Exp. Clin. Endocrinol. Diabetes, 118 (2010) 754-756.

[96] G.A. Laughlin, K.M. Cummins, C.L. Wassel, L.B. Daniels, J.H. Ix, The association of fetuinA with cardiovascular disease mortality in older community-dwelling adults: the Rancho Bernardo study, J. Am. Coll. Cardiol., 59 (2012) 1688-1696.

[97] G.A. Laughlin, E. Barrett-Connor, K.M. Cummins, L.B. Daniels, C.L. Wassel, J.H. Ix, Sexspecific association of fetuin-A with type 2 diabetes in older community-dwelling adults: the Rancho Bernardo study, Diabetes Care, 36 (2013) 1994-2000.

[98] N. Stefan, A. Fritsche, C. Weikert, H. Boeing, H.G. Joost, H.U. Häring, M.B. Schulze, Plasma fetuin-A levels and the risk of type 2 diabetes, Diabetes, 57 (2008) 2762-2767.

[99] S.T. Mathews, S. Rakhade, X. Zhou, G.C. Parker, D.V. Coscina, G. Grunberger, Fetuin-null mice are protected against obesity and insulin resistance associated with aging, Biochem. Biophys. Res. Commun., 350 (2006) 437-443.

[100] E.R. Smith, R. Nilforooshan, G. Weaving, N. Tabet, Plasma fetuin-A is associated with the severity of cognitive impairment in mild-to-moderate Alzheimer's disease, J. Alzheimers Dis., 24 (2011) 327-333.

[101] D. Geroldi, P. Minoretti, M. Bianchi, C. Di Vito, M. Reino, M. Bertona, E. Emanuele, Genetic association of alpha2-Heremans-Schmid glycoprotein polymorphism with late-onset Alzheimer's disease in Italians, Neurosci. Lett., 386 (2005) 176-178. 
[102] R. LaClair, K. O'Neal, S. Ofner, M.J. Sosa, C.A. Labarrere, S.M. Moe, Precision of biomarkers to define chronic inflammation in CKD, Am. J. Nephrol., 28 (2008) 808-812.

\section{Figure Legend}

Figure 1. Fetuin-A is affected by multiple biological and behavioral factors. Biological factors include genetics, disease status and aging, among others. Throughout the lifecycle, Fetuin A is highest at infancy and remains stable throughout childhood and adulthood, but there is high variability in circulating values. Several behavioral factors seem to influence Fetuin-A from infancy to aging, but little or no evidence exists their impact during all life stages (italicized words in the grey box). Per example, physical activity, weight loss either by lifestyle change (low-calorie diets) or weight loss surgery, decrease Fetuin-A. On the other hand, excess calorie intake and obesity seem to increase circulating Fetuin-A. At this time, it is unclear how Fetuin-A during infancy is impacted by early life exposures including maternal diet, maternal weight status and breastfeeding. 
Table 1. Challenges to studying human Fetuin-A in vivo

\begin{tabular}{ll}
\hline \multicolumn{1}{c}{ Methodological } & \multicolumn{1}{c}{ Biological } \\
\hline $\begin{array}{l}\text { - Lack of reference values and standard unit } \\
\text { of measure report for Fetuin-A }\end{array}$ & $\begin{array}{l}\text { - Day-to-day and week-to-week variation in } \\
\text { Fetuin-A has not been characterized }\end{array}$ \\
- Multiple commercial ELISA kits exist, but \\
$\begin{array}{l}\text { yield inconsistent values } \\
\text { - Unknown effects of menstrual cycle on } \\
\begin{array}{l}\text { Lack of reporting on control groups and } \\
\text { numerical/absolute values for Fetuin-A }\end{array}\end{array}$ & $\begin{array}{l}\text { - Effect of post-translational modifications } \\
\text { on Fetuin-A function are unclear }\end{array}$ \\
$\begin{array}{l}\text { - Effect of post-translational modifications } \\
\text { on Fetuin-A measurement are unknown } \\
\text { (i.e. glycosylation, phosphorylation) }\end{array}$ & $\begin{array}{l}\text { - Fetuin-A responds to multiple health and } \\
\text { behavioral factors }\end{array}$ \\
\hline
\end{tabular}


Figure 1. Individual Factors that Influence Circulating Fetuin-A

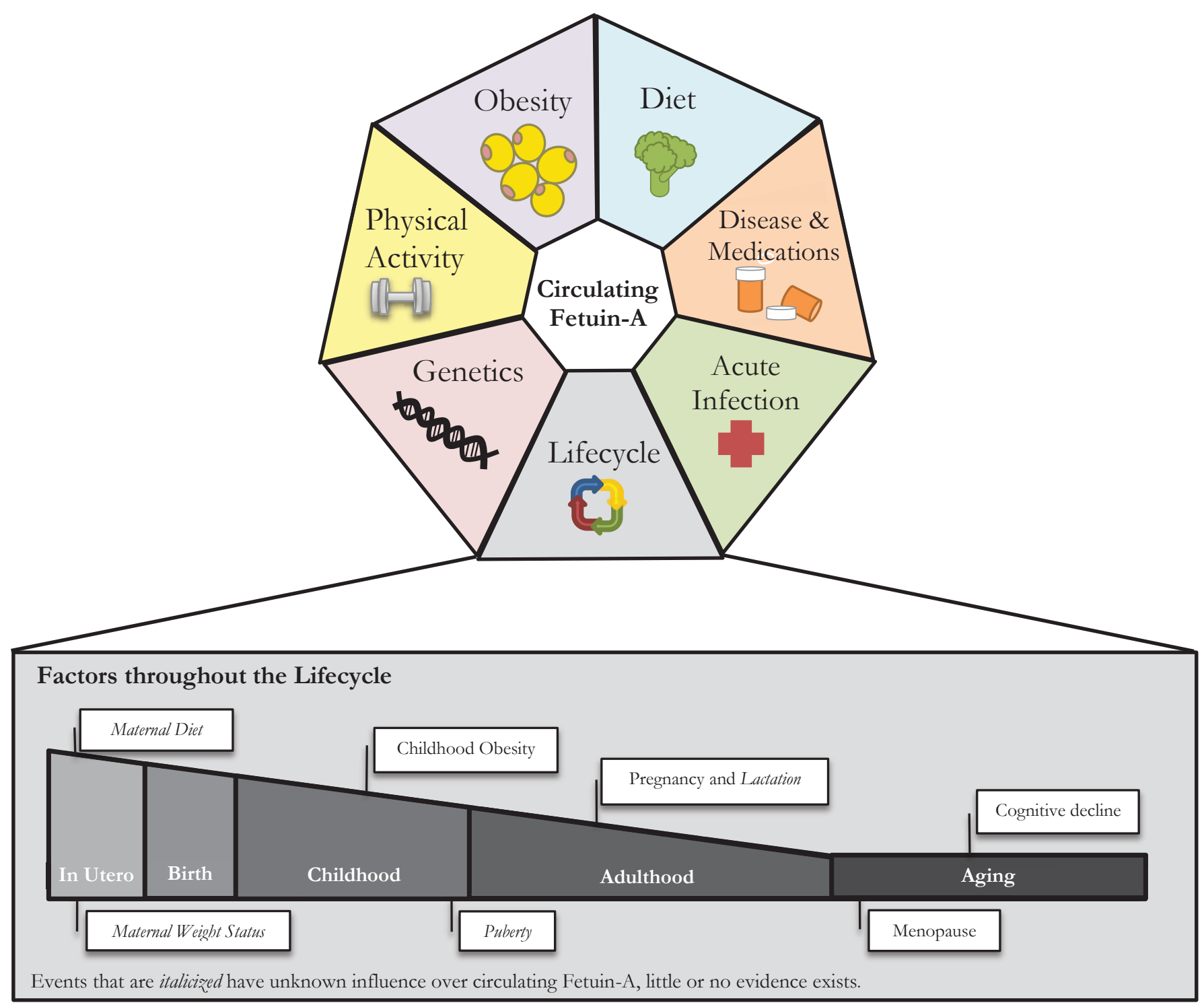

